\title{
Intraocular Surgery in Kyphosis: An Easier Approach
}

\author{
Karanjit S. Kooner $^{a} \quad$ Felise M. Barte ${ }^{b}$ \\ Departments of Ophthalmology at ${ }^{a}$ University of Texas Southwestern Medical Center, and \\ ${ }^{b}$ VA North Texas Health Care System, Dallas, Tex., USA
}

\section{Key Words}

Kyphosis · Intraocular surgery · Intraocular complications

\begin{abstract}
We describe a 49-year-old man with advanced kyphosis and dense cataract, who could only recline to about $40^{\circ}$ from the vertical axis despite a maximal reverse Trendelenburg position and pillows under the head, neck, shoulders and knees. With a single corneal retraction suture at 6 o'clock, the eye could be rotated horizontally, which enabled the surgeon to perform a complex cataract surgery despite prior glaucoma shunt, posterior synechiae, a small pupil and the need to stain the capsule. As the eye can be brought into any desired position with a retraction suture, patients with kyphosis or other conditions that prevent them from assuming a supine position can still have safe intraocular procedures. This maneuver reduces the need to tilt patients to an uncomfortable position that may cause pain, increased breathing difficulty and elevated posterior vitreous pressure.
\end{abstract}

(c) 2013 S. Karger AG, Basel

\section{Introduction}

Kyphosis is a progressive curvature of the thoracic spine, which results in rounding or bowing of the back [1]. A curved spine accompanied by restricted neck motion and poor lung reserve present challenges for an ophthalmic surgeon, who is used to patients' neck and head resting flat on the operating table and the eye in the horizontal position. Intraoperative difficulties include poor view, inability to maneuver surgical instruments properly and constant threat of elevated vitreous pressure. Several innovative solutions have been published, including the maximal reverse Trendelenburg position and pillows under the head and neck [2], use of a donut and wedge-shaped head positioner [3], allowing patients to remain in the upright sitting position while their head is positioned horizontally in a 
modified waiting room chair [4], performing surgery while standing [5, 6], using a 4-section orthopedic operating table [7], or laying the patients on their sides [8]. However, these modifications may still not work in all cases.

\section{Case Presentation}

A 49-year-old obese male with controlled secondary glaucoma needed cataract surgery in the right eye. He had ankylosing spondylitis-related extreme kyphosis and a neck in fixed flexion. His pulmonary reserve was also very low. The past ocular history was positive for recurrent uveitis and glaucoma shunt surgery in the right eye. The pupil was small and fixed from posterior synechia. Cataract density was +4 , and the best-corrected visual acuity was $20 / 200$. The anterior chamber was shallow and the glaucoma shunt tube was visible at the 11 o'clock position. The patient was planned for pupillary dilation using iris retraction hooks, lens capsule staining with trypan blue and phacoemulsification with intraocular implantation under local anesthesia. The anesthesiologist was reluctant to use general anesthesia because of the patient's disability and poor health.

In the operating room, the patient could only recline to about $40^{\circ}$ from the vertical axis despite maximal reverse Trendelenburg position and a number of pillows under his back, neck, head and legs (fig. 1). With the surgeon at the temporal side of the patient, the eye was also seen reclining at $40^{\circ}$ (fig. 2). While trying to focus the microscope, it became evident that by grasping the eye at the inferior limbus with a fine forceps and pulling it upwards, it could be moved to a more desirable horizontal position. A single 7/0 Vicryl ${ }^{\mathrm{TM}}$ (polyglactin) corneal retraction suture was placed at the limbus at the 6 o'clock position to achieve the desired view (fig. 3). The assistant surgeon was able to rotate the eye to the desired positions during the whole procedure by relaxing or pulling the suture. There were no intraocular complications, and the postoperative recovery was uneventful. The final bestcorrected visual acuity was $20 / 30$.

\section{Discussion}

Patients with kyphosis may present several unique challenges to an eye surgeon, the foremost being the inability to lay the head and neck in the ideal horizontal position. The resulting poor view makes focusing and manipulation of tissues and instruments difficult. Tilting the head end of the operating table down and raising the patient's legs may compromise venous return from the orbit, causing venous engorgement and elevated posterior vitreous pressure during surgery. In addition, the patient's poor pulmonary reserve itself may further impede orbital/jugular venous return, resulting in increased posterior vitreous pressure. Other features of kyphosis that may adversely affect intraocular surgery are tenderness and stiffness of the back and difficulty in breathing.

Several practical solutions have been described by very experienced surgeons but complications may still occur. A wedge-shaped pillow with a built-in donut for the positioning of the head has been used by some surgeons [3]. Livingston and Mackool [3] recommended using more than one of these positioners in severe cases. Another approach suggested performing cataract surgery while standing, using loupes and making an inferotemporal scleral tunnel $[5,6]$. This surgery was complicated by a posterior capsular tear requiring anterior vitrectomy. The surgeons were unable to remove the subincisional cortex because of a shallow anterior chamber secondary to elevated posterior vitreous pressure. Poor 
visualization and the unaccustomed standing position may have contributed to the complications. Prasad et al. [7] recommended using a 4-section orthopedic operating table to enable an extreme reverse Trendelenburg position for cataract and glaucoma surgery. However, because of a blurred vision, these authors were unable to perform curvilinear capsulorhexis and had to resort to can-opener capsulotomy. In short, there is no single solution for the different groups of difficult patients.

We have found that a single retraction suture at the inferior limbus can rotate the eye to any desired position. This may allow both the patient and surgeon to be in a comfortable position without compromising good view. It may be tried practically in all patients with kyphosis or other conditions that prevent them from reclining such as torticollis [9], chronic obstructive pulmonary disease, chronic congestive heart failure or morbid obesity. The surgeon can still sit on the preferred temporal side of the patient and perform intraocular surgery of choice. With this maneuver, it may be unnecessary to resort to extreme and uncomfortable tilting of the patient and avoid complications such as those described above.

\section{Acknowledgments}

This study was supported in part by an unrestricted grant from the Society to Prevent Blindness New York, and Visual Science Core Grant EY 020799.

\section{Disclosure Statement}

The authors have no financial or proprietary interest in the materials described in the article.

\section{References}

1 Freeman BL III: Scoliosis and kyphosis; in Canale ST, Beaty JH (eds): Campbell's Operative Orthopaedics (ed 11). Philadelphia, Mosby Elsevier, 2007, chapt 38.

- Gordon ML, Rodriguez AA, Olson MD, Miller KM: Pillow case. J Cataract Refract Surg 2005;31:1824-1825.

-3 Livingston M, Mackool RJ: Donut wedge cataract positioner (letter). J Cataract Refract Surg. 1995;21:5-6.

-4 Fine IH, Hoffman RS, Binstock S: Phacoemulsification performed in a modified waiting room chair. J Cataract Refract Surg 1996;22:1408-1410.

5 Rimmer S, Miller KM: Phacoemulsification in the standing position with loupe magnification and headlamp illumination. J Cataract Refract Surg 1994;20:353-354.

6 Hunter LH: Standing while performing phacoemulsification. J Cataract Refract Surg 1995;21:111.

7 Prasad SP, Kamath GG, Philips RP: Phacoemulsification in a patient with marked cervical kyphosis. J Cataract Refract Surg 2000;26:1258-1260.

-8 Sambandam SPT: Cataract extraction in a humpback woman. Indian J Ophthalmol 1974;22:35, available at http://www.ijo.in/text.asp?1974/22/1/35/31381.

L Liu C: Phacoemulsification in a patient with torticollis (letter). J Cataract Refract Surg 1995;21:364. 


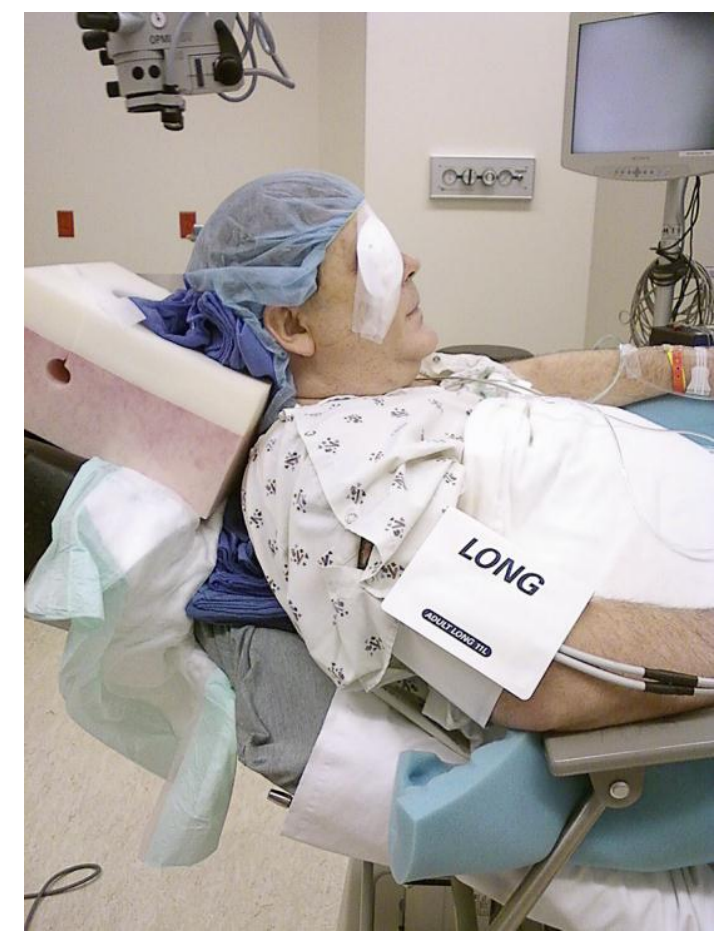

Fig. 1. The patient with kyphosis in the position best tolerated during surgery.

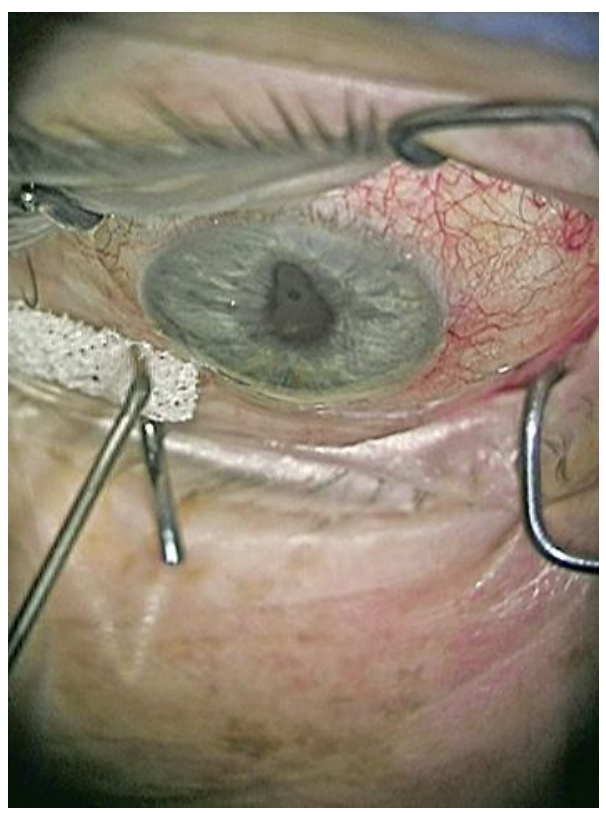

Fig. 2. The virtual vertical position of the eye at the beginning of the surgery. 
Case Reports in

Ophthalmology
Case Rep Ophthalmol 2013;4:34-38

DOI: $10.1159 / 000353227$

Kooner et al.: Intraocular Surgery in Kyphosis: An Easier Approach

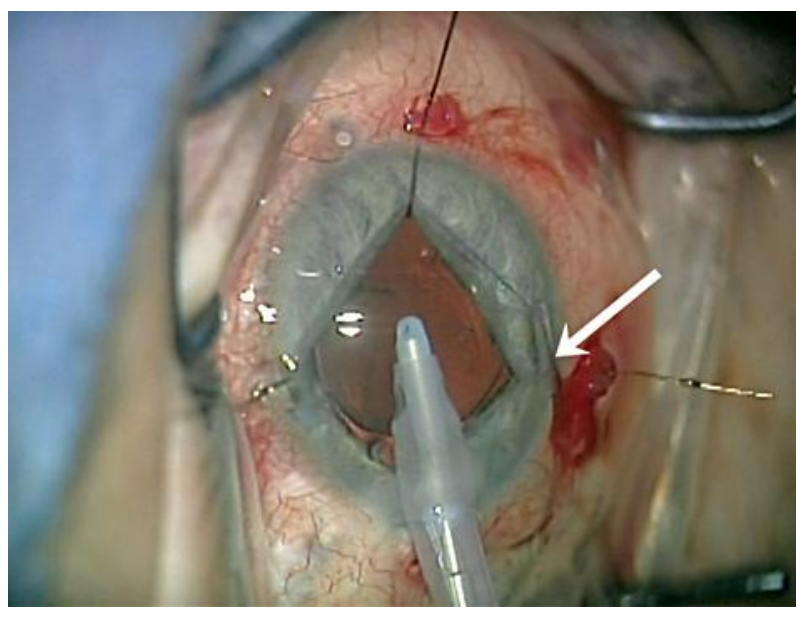

Fig. 3. Pull on the corneal retraction suture (white arrow) moves the eye into a more horizontal position and allows successful completion of cataract surgery. Iris retraction sutures keep the pupil dilated. 